\title{
Natural Killer Cell Lymphoblastic Leukaemia/Lymphoma: Case Report and Review of the Recent Literature
}

\author{
Qanita Sedick $^{\mathrm{a}}$ Sultan Alotaibi ${ }^{\mathrm{b}}$ Saeed Alshieban ${ }^{\mathrm{c}} \quad$ Khalid Ben Naheet $^{\mathrm{a}}$ \\ Ghaleb Elyamany ${ }^{a}$ \\ ${ }^{a}$ Department of Central Military Laboratory and Blood Bank, Prince Sultan Military \\ Medical City, Riyadh, Saudi Arabia; ${ }^{b}$ Department of Adult Hematology, Prince Sultan \\ Military Medical City, Riyadh, Saudi Arabia; ${ }^{C}$ King Abdulaziz Medical City, King Saud bin \\ Abdulaziz University for Health Sciences, Riyadh, Saudi Arabia
}

\section{Keywords}

Natural killer cell lymphoblastic leukaemia/lymphoma · Natural killer cell · Haematopoietic tumour

\begin{abstract}
Natural killer (NK) cell lymphoblastic leukaemia/lymphoma is a rare haemopoietic tumour currently defined in the 2008 WHO classification under the category of acute leukaemias of ambiguous lineage. A diagnosis of this type of leukaemia is considered in cases expressing CD56 along with immature T-cell-associated markers such as CD2 and CD7 with absence of B-cell and myeloid markers; in addition, blastic plasmacytoid dendritic cell leukaemia should be excluded. Prior to 2008, these precursor NK cell lymphoblastic leukaemias/lymphomas were categorized as myeloid/NK cell acute leukaemia with a phenotype identical to acute myeloid leukaemia with minimal differentiation. While the new classification has merit in having excluded myeloid expression, there is still persistent confusion in the literature and on a practical level with regard to precursor NK cell neoplasms. There is a paucity of recent case reports in the literature after the new WHO classification of this neoplasm. Due to the rarity of this neoplasm, an accurate pathological diagnosis is often difficult. In this article, we describe a case of precursor NK cell lymphoblastic leukaemia/lymphoma presenting with
\end{abstract}




\section{Case Reports in Oncology}

Sedick et al.: Natural Killer Cell Lymphoblastic Leukaemia/Lymphoma: Case Report and Review of the Recent Literature

unique morphological features and conflicting immunophenotypes. We also review all case reports of this neoplasm after the WHO 2008 classification.

\section{Introduction}

Precursor natural killer (NK) cell lymphoblastic leukaemia/lymphoma is a rare haemopoietic tumour currently defined in the $2008 \mathrm{WHO}$ classification under the category of acute leukaemias of ambiguous lineage. A diagnosis of this leukaemia is considered in cases expressing CD56 along with T-cell-associated markers such as CD2 and CD7 with absence of B-cell and myeloid markers. The T-cell and immunoglobulin (Ig) receptor genes must be in the germline configuration, and blastic plasmacytoid dendritic cell leukaemia should be excluded $[1,2]$.

Due to the rarity of this neoplasm, an accurate pathological diagnosis is often difficult. In addition, early NK cell progenitors express no markers or may express markers which overlap with early T-cell markers including CD7, CD2, CD5, and cytoplasmic CD3. Therefore distinguishing between T-cell acute lymphoblastic leukaemia (T-ALL) and NK cell tumour may be difficult. More specific markers of NK cell progenitors, such as CD94 and CD161, are not commonly tested [1].

In this article, we describe a case of precursor NK cell lymphoblastic leukaemia/lymphoma presenting with unique morphological features and conflicting immunophenotypes. We also review all case reports of this neoplasm after the WHO 2008 classification.

\section{Case History}

A 19-year-old male patient of Saudi Arabian origin presented with extensive cervical and axillary lymphadenopathy with no previous history of illness or medications. Apart from lymphadenopathy, there were no relevant findings on clinical examination. There was no evidence of skin lesions.

A CT scan revealed bilateral multiple matted cervical and supraclavicular lymphadenopathy. Fullness of the nasopharynx with enhancing soft tissue and prominent palatine and lingual tonsils were identified. There were prominent mediastinal lymph nodes and enlargement of the thyroid gland. Some abdominal, pelvic, and inguinal lymphadenopathy was present. The liver was enlarged, measuring $18 \mathrm{~cm}$ (mid-clavicular line). The spleen was of borderline size. A PET scan agreed with these findings, showing extensive hypermetabolic lymphomatous masses involving the bilateral cervical and supraclavicular regions as well as the mediastinal and axillary regions with some abdominal lymphadenopathy noted (Fig. 1).

The initial complete blood count showed a haemoglobin level of $13.7 \mathrm{~g} / \mathrm{dL}$, a total leukocyte count of $2.2 \times 10^{9} / \mathrm{L}$, and a platelet count of $286 \times 10^{9} / \mathrm{L}$. A peripheral blood smear showed a leucoerythroblastic reaction with occasional blasts (approx. 3\%). CSF examination showed no blasts.

A cervical lymph node biopsy was performed, and it showed infiltration of medium-tolarge malignant cells. Immunohistochemical stains revealed positive CD3 (partial), CD7, TdT, CD2, CD43, and bcl-2, whereas CD4, CD8, CD5, CD20, Pax5, CD79a, bcl-6, MPO, and other B-cell and myeloid markers were negative (Fig. 2). Epstein-Barr virus-encoded small RNA in situ hybridization for Epstein-Barr virus was negative. 


\section{Case Reports in Oncology}

Based on partial expression of CD3 and negativity to MPO and CD79a in addition to positivity to CD2, CD7, and TdT, a histological diagnosis of T-ALL was made on lymph node biopsy. This initial panel, however, failed to include CD56, and thus an NK cell neoplasm was not considered at this point.

Staging bone marrow (BM) aspirate was taken and a trephine biopsy was performed. The BM showed extensive infiltration of immature mononuclear cells, i.e., around $47 \%$ of the myelogram. These cells were intermediate to large in size, with variable cytoplasm and vesicular chromatin. The majority of the cells displayed nuclear indentation or clefting. Normal trilineage haemopoiesis was decreased and demonstrated dysplastic changes mainly in the granulocytic lineage (Fig. 3).

A flow cytometric analysis was done on the BM aspirate using CD45/side scatter gating; a population of blasts expressed dimmer CD45, HLA-DR, CD16/56 (on \% of the population), CD2, CD7, CD34, and CD38. A subpopulation expressed cytoplasmic CD3 (20\%) and CD117 $(10 \%)$. The B-cell markers and other myeloid markers were negative. Of note, CD4, CD8, and CD5 were negative in the blast population (Fig. 4). Immunohistochemical staining of the trephine biopsy confirmed the flow cytometric findings. PCR analysis revealed a germline configuration of the Ig heavy chain (IgH) and T-cell receptor (TCR) genes.

Based on these BM findings, a diagnosis of acute leukaemia of ambiguous lineage according to the WHO classification (2008) or T-ALL was considered. The clear expression of CD56 in our population with the absence of IgH and TCR rearrangements, in addition to the absence of CD4 expression and no skin lesions clinically, led us to a diagnosis of precursor NK cell lymphoblastic leukaemia/lymphoma.

A cytogenetic evaluation showed no specific recurrent genetic abnormalities for ALL; however, there was a tumour clone with translocation between the short arm of chromosome 1 at p36.1 and the long arm of chromosome 14 at q24 and isochromosome $17 \mathrm{q}$ as a clonal evolution. FISH analysis confirmed that the TP53 gene was deleted in $14 \%$ of the studied cells and was negative for ALL panel-specific abnormalities; the cytogenetic result was as follows: $46, \mathrm{XY}, \mathrm{t}(1 ; 14)(\mathrm{p} 36.1 ; \mathrm{q} 24)[3] / 46, \mathrm{XY}$,idem,i(17)(q10)[2]/46,XY[5]. nuc ish(ABL1, BCR)x2[100]/(ETV6,RUNX1)x2[100]/(5'MLL,3'MLL, $5^{\prime}$ MLL con $3^{\prime}$ MLL)x2[100]/(5'MYC, 3'MYC,5'MYC con 3'MYC)x2[100]/(TCF3,PBX1)x2[100]/(TP53x1)[28/200].

The patient was treated using the augmented Berlin-Frankfurt-Münster chemotherapeutic protocol with prednisolone and 4 weekly cycles of daunorubicin with vincristine along with L-asparaginase. On day 14 the BM showed less than 5\% blasts, and on day 28 the BM was in remission.

The patient received consolidation chemotherapy. He was provided with interim maintenance II and delayed intensification II. CSF analyses for CNS disease remained negative during the whole course of therapy, and he received his intrathecal methotrexate doses. He has been started on maintenance chemotherapy after consolidation and continues to be in remission.

\section{Discussion}

In 2008, the WHO attempted to redefine precursor NK cell lymphoblastic leukaemia/lymphoma based on the expression of CD56 with the immature T-cell markers CD7, CD2, and cytoplasmic CD3 and the absence of B-cell markers and myeloid markers. Early NK cell progenitors express T-cell markers such as CD2, CD7, CD5, and cytoplasmic CD3, which 


\section{Case Reports in Oncology}

makes the distinction between T-ALL and NK cell neoplasms rather difficult. Neural cell adhesion marker CD56 expression is more characteristic of NK cells, but it does not exclude T-ALL [1].

There is a paucity of recent data (after the 2008 WHO reclassification) and case reports of precursor NK cell lymphoblastic leukaemia/lymphoma in the literature. The most recent case report documented is on a 24-year-old female patient of Greek origin who presented with neurological symptoms of vertigo and unsteady gait. MRI revealed several lesions in the pons and cerebellar peduncle. A biopsy showed infiltration of intermediate-sized agranular lymphocytes expressing TdT and CD56 with absence of all myeloid, B-cell, and T-cell markers. An assessment of TCR and IgH gene rearrangement resulted negative, and based on these findings the diagnosis of precursor NK cell lymphoblastic leukaemia/lymphoma was made [2].

Another recent case report is on a 24-year-old Indian male patient presenting with a 1month history of fever, malaise, and cervical and axillary lymphadenopathy. The patient had 91\% blasts in the peripheral blood which expressed CD56 and the immature T-cell markers CD7 and CD2 with absence of all myeloid, B-cell, and other T-cell markers. The diagnosis of precursor NK cell lymphoblastic leukaemia/lymphoma was made [3].

Our case, in particular, is of interest due to the granulocytic dysplasia identified on morphological examination of the BM. There was no history of a previous malignancy, chemotherapy, drug intake, or haematinic deficiencies (such as vitamin $B_{12}$ or red cell folate) which may have obscured this finding. We performed a literature review, and to the best of our knowledge this finding has not been previously reported. In 2003, 3 cases of blastic NK cell lymphoma associated with myelodysplasia and myelomonocytic leukaemia were reported [4]. Similarly, some cases are reported as acute leukaemias possibly arising from of immature NK cell neoplasms expressing CD56 along with different combinations of myeloid antigens [5-8]. According to the 2008 WHO classification, all of the above-mentioned cases will be diagnosed as AML expressing NK cell markers.

In our case study, based on these BM findings, a diagnosis of acute leukaemia of ambiguous lineage according to the WHO classification of 2008 or T-ALL was considered. In our differential diagnosis, we considered T-ALL, precursor NK cell lymphoblastic leukaemia/lymphoma, mixed-phenotype acute leukaemia not otherwise specified, and blastic plasmacytoid dendritic cell leukaemia. The absence of clear-cut B-cell and myeloid markers excluded mixed-phenotype acute leukaemia from our initial differential diagnosis. Due to partial expression of cytoplasmic CD3, T-ALL was considered. Although cytoplasmic CD3 is lineage specific for T cells, it can be positive in NK cell neoplasms. The clear expression of CD56 in our population made a diagnosis of precursor NK cell lymphoblastic leukaemia/lymphoma more likely. We excluded blastic plasmacytoid dendritic cell leukaemia based on the absence of CD4 expression and as there were no skin lesions clinically. Exclusion of this neoplasm is required by the WHO for diagnosing precursor NK cell lymphoblastic leukaemia/lymphoma. Furthermore, the absence of IgH and TCR rearrangements following PCR analysis consolidated our diagnosis of precursor NK cell lymphoblastic leukaemia/lymphoma.

\section{Conclusion}

Our case highlights the diagnostic difficulties regarding the rare entity of precursor NK cell lymphoblastic leukaemia/lymphoma. There is a paucity of recent case reports in the 
Sedick et al.: Natural Killer Cell Lymphoblastic Leukaemia/Lymphoma: Case Report and Review of the Recent Literature

literature after the 2008 WHO classification of this neoplasm. Despite this, the limited data in addition to our case report indicate diversity in the clinical presentation, immunophenotyping, and histological presentation of this neoplasm. The granulocytic dysplasia in conjunction with the clonality of NK cells is of interest in our report, and may give additional insight into the pathogenesis of this neoplasm. This highlights the urgent need for further research and case documentation on precursor NK cell lymphoblastic leukaemia/lymphoma. There is also a need to further validate more specific early NK markers, such as antibodies against killercell immunoglobulin-like receptors, to narrow down the diagnostic criteria as well as for a more specific targeted therapy and better clinical outcome.

\section{Statement of Ethics}

Informed consent was obtained and the case study has been approved by the department's committee on human research.

\section{Disclosure Statement}

The authors have no funding or conflicts of interest to disclose.

\section{References}

1 Swerdlow SH, Campo E, Harris NL, Jaffe ES, Pileri SA, Stein H, Thiele J, Vardiman JW: WHO Classification of Tumours of Hematopoietic and Lymphoid Tissues. Lyon, IARC, 2008.

-2 Kontogeorgi E, Papadopoulos D, Zafeiropoulou E, Karagkounis G, Argyrakos T, Stranjalis G, Rontogianni D, Karakalos D: A 24 year-old woman with relapsing brainstem manifestations and multiple focal brain lesions. Natural killer cell lymphoblastic lymphoma/leukemia. Brain Pathol 2015;25:115-116.

-3 Jain S, Kumar R, Purohit A, Pati HP: Precursor NK cell lymphoblastic leukemia/lymphoma - report of a case with literature review. Indian J Hematol Blood Transfus 2014;30(suppl 1):283-285.

4 Kazakov DV, Mentzel T, Burg G, Dummer R, Kempf W: Blastic natural killer-cell lymphoma of the skin associated with myelodysplastic syndrome or myelogenous leukaemia: a coincidence or more? $\mathrm{Br} \mathrm{J}$ Dermatol 2003;149:869-876.

5 Chen B, Xu X, Ji M, Lin G: Myeloid/NK cell acute leukemia. Int J Hematol 2009;89:365-367.

-6 Ma Y, Chen B, Xu X, Lin G: Myeloid/natural killer cell precursor acute leukemia with multiple subcutaneous nodules as the initial presentation: a case report and literature review. Int J Hematol 2009;90:243-247.

-7 Hashii Y, Okuda T, Ohta H, Ozono K, Hara J: Pediatric myeloid/NK cell precursor lymphoma/leukemia expressing T/NK immunophenotype markers. Int J Hematol 2010;91:525-529.

-8 Guan XQ Xu L, Ke ZY, Huang LB, Zhang XL, Zhang YC, Luo XQ: Five Chinese pediatric patients with leukemias possibly arising from immature natural killer cells: clinical features and courses. Pediatr Hematol Oncol 2011;28:187-193. 


\section{Case Reports in Oncology}

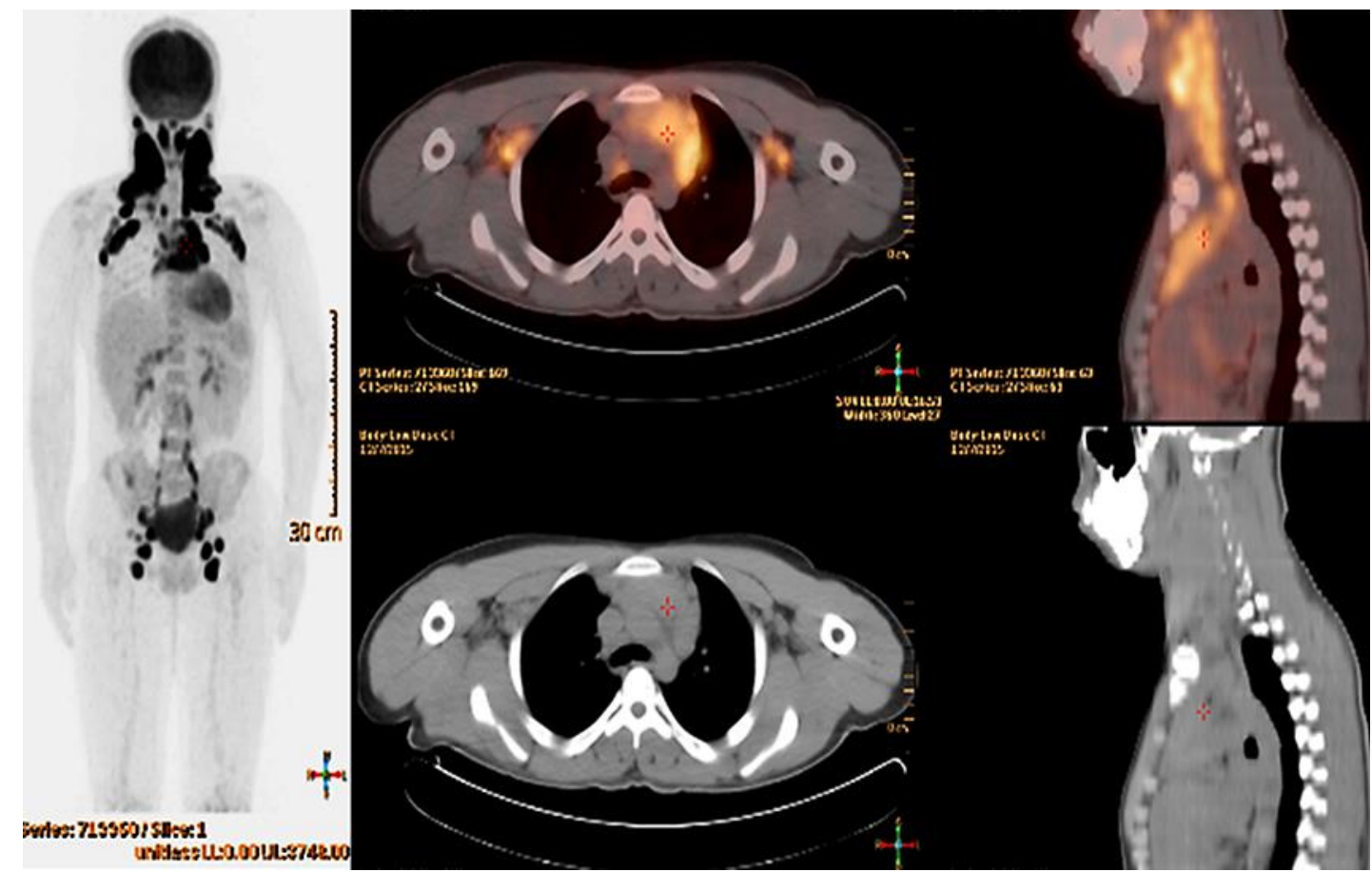

Fig. 1. ${ }^{18}$ F-fluorodeoxyglucose PET/CT showing extensive hypermetabolic masses involving the bilateral cervical and supraclavicular regions, as well as enlarged hypermetabolic palatine tonsils. Mediastinal and bilateral hypermetabolic axillary, pelvic, inguinal, and, to a lesser extent, abdominal lymphadenopathy is visible. 


\section{Case Reports in Oncology}
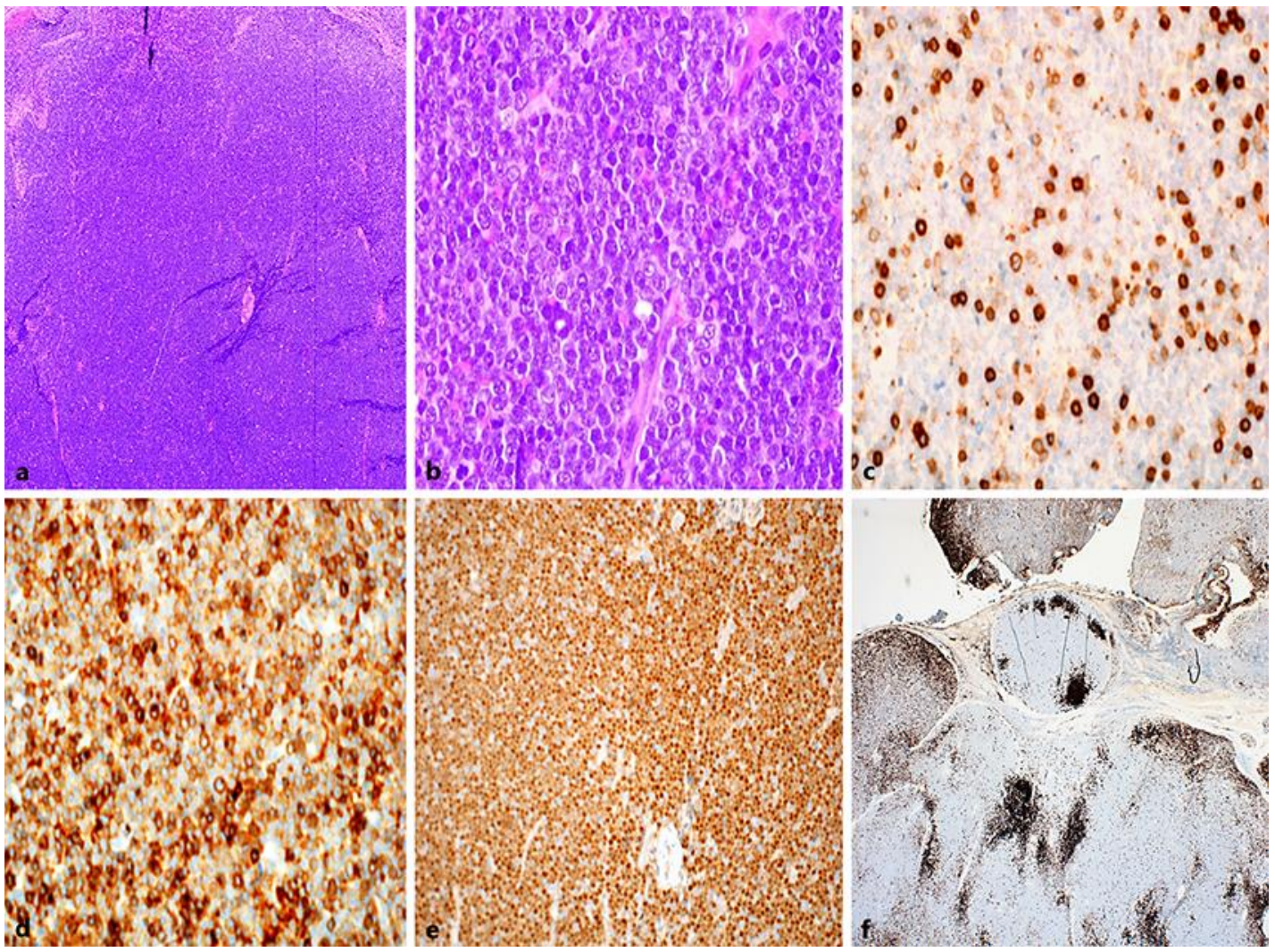

Fig. 2. Low- (a) and high-power (b) images of a haematoxylin-eosin-stained cervical lymph node biopsy showing effacement of the lymph node architecture by medium-to-large cells with a round, oval, or irregular nuclear contour, vesicular nuclei with fine chromatin, and one or more nucleoli. The immunohistochemical stains show that the neoplastic cells are positive for CD3 (partial) (c), CD7 (d), and TdT (e) and negative for CD20 (f).
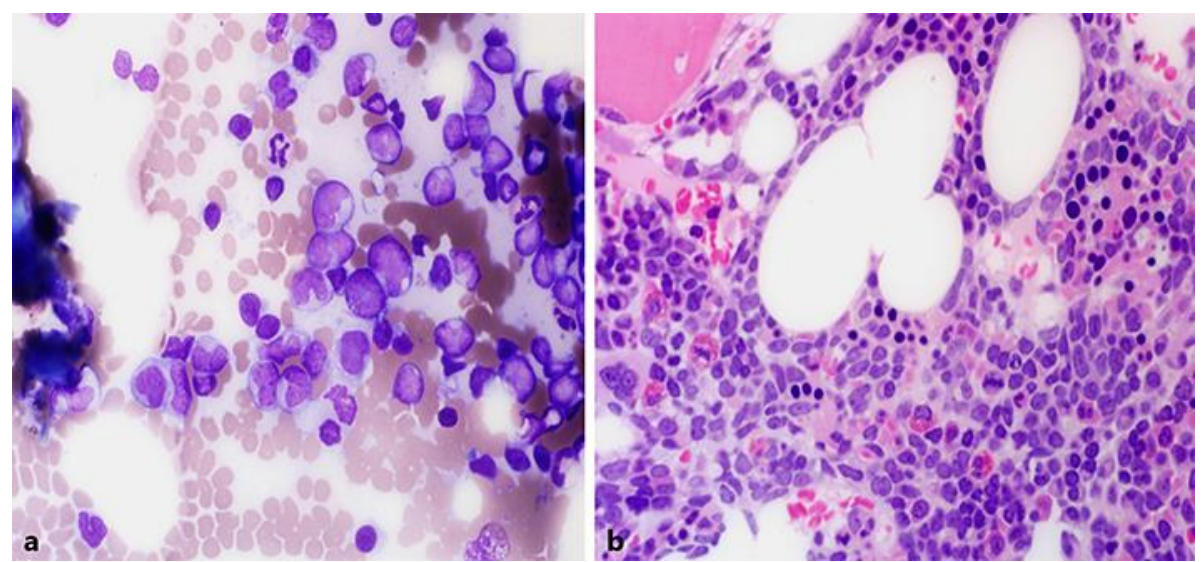

Fig. 3. Bone marrow aspirate (a) and trephine biopsy (b) showing an infiltration of immature mononuclear cells. These cells are intermediate to large in size, with variable cytoplasm and vesicular chromatin. The majority of the cells display nuclear indentation or clefting. 

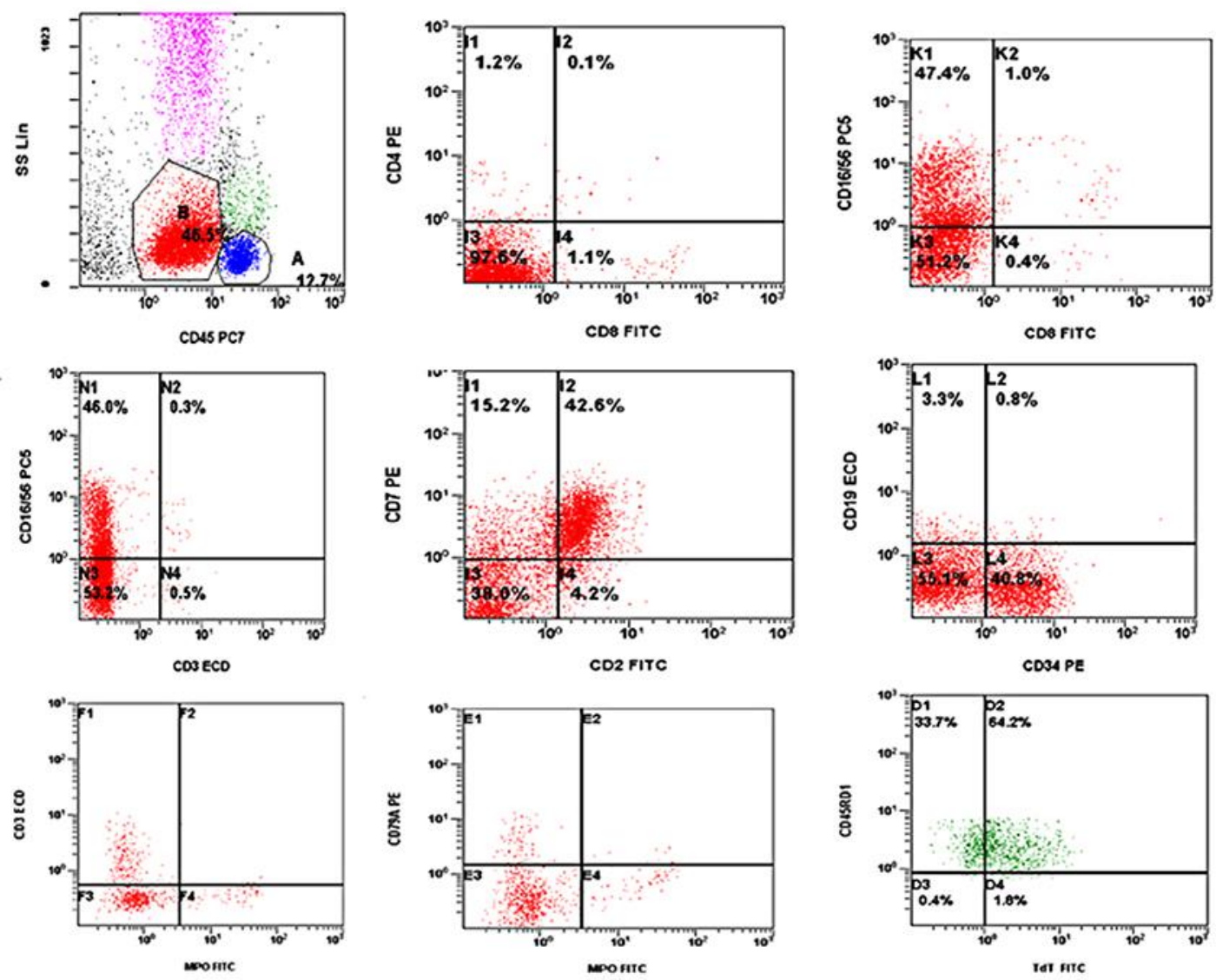

Fig. 4. Flow cytometric analysis of bone marrow aspirate using CD45/side scatter gating. It shows a population of blast cells (47.5\%) expressing CD45, CD16/56, CD2, CD7, and CD34 more dimly, and negative for surface CD3 and CD19. Cytoplasmic staining shows a subpopulation expressing cytoplasmic CD3 (20\%) and positive for TdT and negative for CD79a and MPO. 